\title{
Patogénesis de la pudrición blanda de la lechuga (Lactuca sativa L.) en la Sabana de Bogotá causada por Sclerotinia sclerotiorum (Lib.) de Bary y Sclerotinia minor Jagger. Una revisión
}

\section{Pathogenesis of soft rot in lettuce (Lactuca sativa L.) in the Bogota Plateau caused by Sclerotinia sclerotiorum (Lib.) de Bary and Sclerotinia minor Jagger. A review}

SILVIA LILIAM PÉREZ S. ${ }^{1}$

WILSON PIEDRAHÍTA C.2,3

GERMÁN ARBELÁEZ²

Daño por Sclerotinia en lechuga en la Sabana de Bogotá.

Foto: W. Piedrahita C.

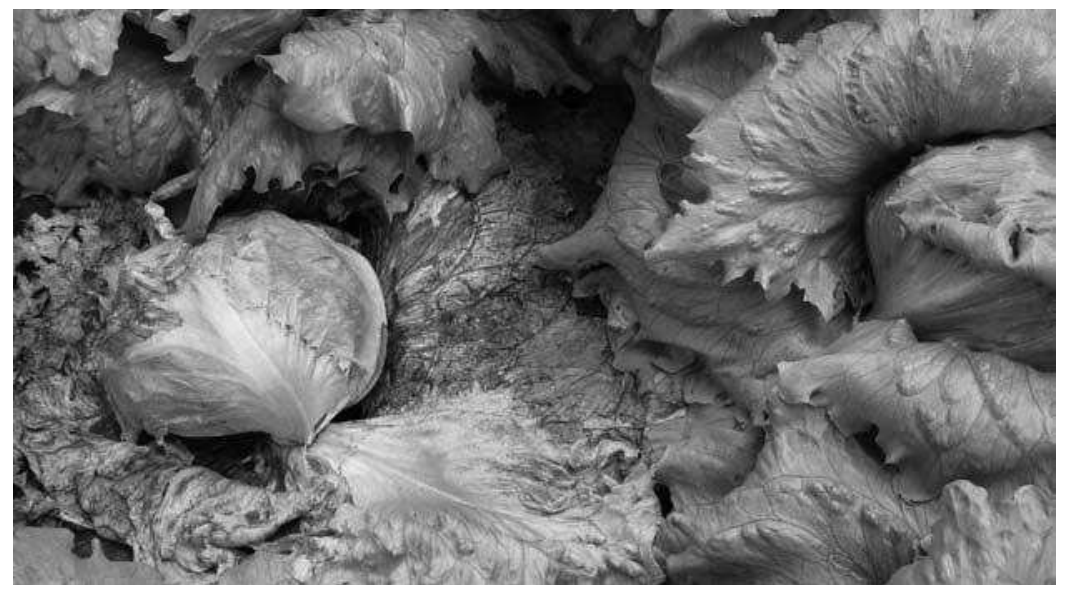

\section{RESUMEN}

La pudrición blanda, causada por Sclerotinia sclerotiorum y S. minor, es la enfermedad más limitante en Colombia y ocasiona pérdidas económicas en la lechuga equivalentes a una reducción entre el 30-50\% de la población de plantas. Tanto las condiciones climáticas como la densidad de los esclerocios en el suelo influencian los niveles de pérdidas en el cultivo, siendo por lo tanto esta enfermedad de gran importancia para el horticultor. Para diseñar una estrategia de manejo de la enfermedad, se requiere conocer el agente causal, su biología, los factores internos y externos que controlan la infección y la expansión de la enfermedad. El presente trabajo es una revisión bibliográfica que resume la amplia información publicada sobre estos dos agentes causantes de la enfermedad pudrición blanda. Se revisan algunos aspectos biológicos de los agentes causales, el proceso de la patogénesis, los requerimientos nutricionales para el desarrollo de la infección, el papel del ácido oxálico en la patogénesis y la sintomatología de la enfermedad.

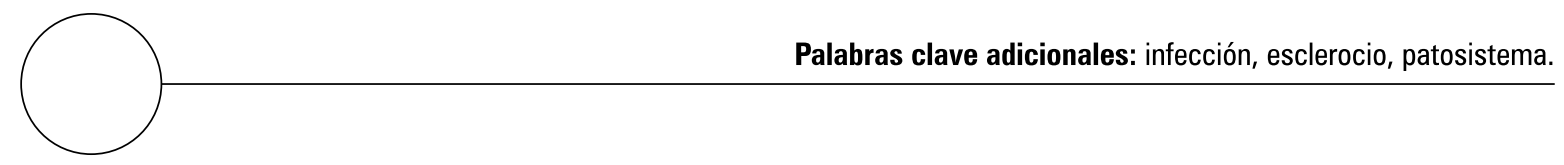

\footnotetext{
Programa de Investigación y Desarrollo, Ball Colombia, Bogotá (Colombia).

Departamento de Agronomía, Facultad de Agronomía, Universidad Nacional de Colombia, Bogotá (Colombia).

Autor para correspondencia.wdpiedrahitac@bt.unal.edu.co
} 


\section{ABSTRACT}

The soft rot caused by Sclerotinia sclerotiorum and S. minor is the most limiting disease in Colombia and results in economic losses in lettuce between $30-50 \%$ of plant density; both climatic conditions and sclerotia density in soil influence levels of crop losses, being so this disease is of great importance for the farmer. In order to design a strategy for managing the disease, it is required knowledge of the causal agent, its biology, internal and external factors that control the infection and the spread of the disease. This study is a bibliographic review, which summarizes the extensive information published on these two causal agents of the soft rot disease. The biological aspects of causal agents, pathogenesis process, the nutritional requirements for the development of infection, the role of oxalic acid in the pathogenesis, and symptoms of the disease are reviewed.

Additional key words: infection, sclerotia, pathosystem.

Fecha de recepción: 27-08-2009

Aprobado para publicación: 30-11-2009

INTRODUCCIÓN

La pudrición blanda es una enfermedad común en el cultivo de la lechuga en la Sabana de Bogotá y es causante de pérdidas estimadas entre el 30 y $50 \%$ de la población de plantas (Arias y Tautiva, 2006). En una evaluación de la enfermedad realizada por Martínez-Pérez (2008) en los municipios de Funza y Mosquera, Cundinamarca, se encontró una incidencia de 32 y $52 \%$ y una pérdida final de plantas de 17 y $16 \%$ respectivamente pero con una población de un esclerocio por muestra de suelo; Smith (2007) en un estudio realizado para caracterizar el moho blanco de la lechuga en Cota, Cundinamarca, encontró una incidencia del $42 \%$ con 25 esclerocios por $1.000 \mathrm{~g}$ de suelo en el testigo absoluto; por su parte, Ávila de Moreno et al. (1996) catalogan la enfermedad como una de las más destructivas y frecuentes en muchas plantas suculentas, causante de Damping-off en semilleros y pudrición en plantas adultas en el campo y durante el almacenamiento. En España, la enfermedad se presenta con alto grado de incidencia, lo cual unido a la falta de medios efectivos de control, principalmente químicos, la convierten en la principal causante de pérdidas del cultivos (Campos et al., 1998). De igual forma Sclerotinia se encuentra reportado causando importantes pérdidas económicas en diferentes cultivos hortícolas intensivos y extensivos como pimentón, papa, fríjol, girasol, soya, canola, colza, entre otros; por ello, estos hongos tienen una distribución amplia en numerosos países (Ren et al., 2007).

\section{ETIOLOGÍA DE LA ENFERMEDAD}

Los agentes causales de la Pudrición Blanda o Moho Blanco de la lechuga son los hongos Sclerotinia sclerotiorum (Lib.) de Bary y Sclerotinia minor Jagger (Steadman, 1979; Subbarao et al., 1997; Subbarao, 1998; Koike y Davis, 2009; Laemmlen, 2003). En una evaluación realizada en sendos cultivos de lechuga en Mosquera y Funza (Cundinamarca) se encontraron $S$. minor y $S$. sclerotiorum respectivamente en el área de estudio (Martínez-Pérez, 2008). Por otra parte, la investigación realizada sobre métodos de control de pudrición blanda en Mosquera (Cundinamarca) por Arias y Tautiva (2006) mostró que el inóculo correspondía a $S$. sclerotiorum. De igual forma, en el municipio de Cota (Cundinamarca) en un estudio de control por medio de solarización, Gil et al. 
(2009) encontraron que la especie dominante fue S. minor. Lo anterior muestra que ambas especies han sido reportadas en la Sabana de Bogotá. Estos hongos se pueden diferenciar en el campo por el tamaño y la forma de los esclerocios y en laboratorio a través de zimogramas de las enzimas pécticas extracelulares (Cruickshank, 1983).

\section{CICLO DE VIDA DE LOS AGENTES CAUSALES}

\section{Sclerotinia sclerotiorum}

El ciclo de vida de S. sclerotiorum tiene dos fases. La primera, representada por formas de resistencia denominadas esclerocios, corresponde al $90 \%$ del total, y en esta puede permanecer semanas o años para volverse a activar (Ferreira y Boyle, 1992; Adams y Ayers, 1979), pero requiere para ello de condiciones ambientales apropiadas de humedad (Ferreira y Boyle, 1992 y Laemmlen, 2003), y adicionalmente precisan nutrientes exógenos promotores de la "germinación" (Ferreira y Boyle, 1992). Los esclerocios son estructuras de resistencia, de forma variable, tipo tubérculo, ricas en nutrimentos y formadas por múltiples hifas; poseen una zona endurecida, formada por una capa continua de puntas de hifas, y seguida en algunos casos por una corteza delgada donde se acumulan las reservas nutritivas, luego sigue una médula amplia formada por hifas entretejidas que representa la parte principal de la estructura que contiene reservas nutritivas intracelulares en las hifas y extracelulares en una matriz continua o discontinua (Willetts y Bullock, 1992).

En las hifas de los esclerocios de Sclerotium rolfsii las paredes contienen el pigmento melanina que no se encuentra en las hifas normales del hongo; presentan además diez aminoácidos en las paredes excepto L-argenina, L-prolina y L-serina que pueden influir en la resistencia a la degradación biológica y química en el suelo (Chet et al., 1967). Es muy probable que los hongos patogénicos incrementen los niveles de $\beta$-caroteno en los esclerocios para contrarrestar los efectos de los procesos oxidativos (Zervoudakis et al., 2003). El tiempo para producir esclerocios está determinado por factores endógenos pero la germinación está condicionada por la temperatura y la intensidad lumínica (Trevethick y Cooke, 1973). Los esclerocios de $S$. sclerotiorum son de mayor tamaño que los de $S$. minor y se diferencian entre sí por los patrones de crecimiento de los micelios siendo en este último menos frecuente la coalescencia de las hifas en esclerocios (Willetts y Wong, 1971). Las hifas formadas a partir de los esclerocios presentan anastomosis, fenómeno que permite el intercambio por fusión entre ellas (Erental et al., 2008).

La segunda fase se inicia cuando los esclerocios "germinan eruptivamente" formando micelio o carpogénicamente hablando estructuras delgadas que terminan en un pequeño apotecio (Purdy, 1979; Abawi y Grogan, 1979; Adams y Ayers, 1979; Ferreira y Boyle, 1992; Walker, 1959; Agrios, 2005; Adams y Tate, 1975; Laemmlen, 2003; Koike y Davis, 2009) con forma de copa o disco de un diámetro de 5 a $15 \mathrm{~mm}$, y en el cual se desarrollan las ascas y ascosporas (Agrios, 2005) para lo cual se requieren condiciones frescas y húmedas (Koike y Davis, 2009). Posteriormente, el micelio formado a partir del esclerocio infecta los tejidos huéspedes vivos (Ferreira y Boyle, 1992; Purdy, 1979; Abawi y Grogan, 1979, Sun y Yang, 2000; Ferreira y Boyle, 1992; Agrios, 2005). La infección causada por los esclerocios puede ocurrir en la superficie del suelo o debajo de esta (Ferreira y Boyle, 1992).

Por otra parte, las ascosporas formadas en el apotecio se liberan profusamente para infectar otras plantas del cultivo. En un campo de lechuga, la dispersión se dio por el viento a cultivos vecinos en un periodo de 2 a 3 semanas y se dispersaron a cultivos adyacentes (Subbarao, 1998; Adams y Ayers, 1979; Laemmlen, 2003; Koike y Davis, 2009). En papa se ha encontrado que las ascosporas provenientes de campos vecinos se constituyen en una importante fuente de infección e 
inhabilitan las medidas de biocontrol, a través de Coniothyrium minitans (Hammond et al., 2008).

Bajo adecuada humedad, la germinación de las ascosporas ocurre entre 3 y $6 \mathrm{~h}$ después de la liberación (Ferreira y Boyle, 1992); pero Subbarao (1998) reporta que la germinación, en presencia de agua en la hoja, se presenta durante 48 h o más. En contacto con la superficie del huésped, el micelio originado por las ascosporas produce apresorios y la penetración de la cutícula se logra por presión mecánica (Boyle, 1921). El tipo de apresorio formado está relacionado con la superficie de contacto: se ha encontrado que en el caso de la lechuga, frijol y colza se forman apresorios simples mientras que otras plantas promueven tipos complejos (Tariq y Jeffries, 1984). Bajo condiciones de laboratorio, Clarkson et al. (2003) encontraron liberación de esporas en apotecios a partir de los $15^{\circ} \mathrm{C}$, sin embargo, la temperatura y la humedad relativa alta afectan la supervivencia de las esporas pero no la viabilidad en presencia de diferentes regímenes lumínicos.

Una vez que el hongo se encuentra dentro de la cutícula causa una disolución enzimática de la pared celular (Walker, 1957), lo cual facilita el desarrollo progresivo sobre la superficie del tejido afectado y la invasión subsiguiente a otros (Ferreira y Boyle, 1992). Esta disolución se realiza a través de sustancias pécticas; así por ejemplo, en coleóptilos de soya infectados con $S$. sclerotiorum se han aislado las isoenzimas endopoligalacturonasa (PG I y PG IV) responsables de la hidrólisis del ácido poligalacturónico y que estimulan en forma diferente la producción de fitoalexinas (Favaron et al., 1992). También existen enzimas diferentes a las pécticas que contribuyen a la penetración al huésped; en el caso de fríjol, Tariq y Jeffries (1987), a través de técnicas ultracitoquímicas, encontraron actividad de una enzima lipolítica en la zona de contacto íntimo entre el apresorio y la superficie foliar.

Posteriormente, se forman esclerocios sobre el tejido muerto (Koike y Davis, 2009; Agrios, 2005).
Los esclerocios retornan al suelo para un periodo de reposo (Adams y Ayers, 1979; Ferreira y Boyle, 1992), que varía de semanas o años antes de que vuelvan a ser activos, para lo cual se requieren condiciones ambientales apropiadas (Laemmlen, 2003). Los esclerocios de S. sclerotiorum son más grandes (2 a $10 \mathrm{~mm}$ de diámetro) que los de S. minor, tienen superficie lisa y son redondeados. Los esclerocios de $S$. minor son pequeños $(0,5$ a $2,0 \mathrm{~mm}$ de diámetro), rugosos, angulares y más numerosos que los de S. sclerotiorum (Laemmlen, 2003).

Patsoukis y Georgiou (2007) consideran que el proceso de desarrollo de los esclerocios en hongos está afectado negativamente por el estrés oxidativo, es decir, el estado tio redox; según los autores, cualquier factor nutricional o no nutricional que inhiba o estimule el estrés oxidativo estimula o inhibe el proceso de biogénesis del esclerocio.

\section{Sclerotinia minor}

En el campo, los esclerocios de $S$. minor rara vez producen apotecios (Imolehin et al., 1980; Laemmlen, 2003) y por tanto las infecciones son causadas directamente por la "germinación" eruptiva de aquéllos (Abawi y Grogan, 1979; Dillard y Grogan, 1985; Patterson y Grogan, 1988). Sin embargo, en condiciones de laboratorio se ha encontrado producción de apotecios por S. minor (Jagger, 1920, cit. por Abawi y Grogan, 1979; Imolehin et al., 1980).

Después de un periodo de latencia, los esclerocios situados a $2 \mathrm{~cm}$ de la raíz principal y $8 \mathrm{~cm}$ de la superficie del suelo desarrollan, en un medio húmedo, una o más protuberancias que crecen y finalmente se rompen liberando una masa de micelio denso (Imolehin et al., 1980). En algunos casos, estas formaciones se reúnen y tales masas de micelio crecen desde los esclerocios a distancias de 2 a $3 \mathrm{~mm}$, infectando raíces cercanas, tallos y hojas senescentes (Subbarao, 1998); sin embargo, Koike y Davis (2009) sostienen que 
los esclerocios de S. minor solo infectan tallos y hojas en contacto con el suelo.

Finalmente cuando la infección ha avanzado, se forma un gran número de esclerocios en la corona y en las partes subterráneas de las plantas infectadas de lechuga, los cuales retornan al suelo cuando los residuos de la cosecha se incorporan en la preparación del mismo (Subbarao, 1998).

Zervoudakis et al. (2003) considerando la hipótesis de la relación entre procesos de diferenciación en hongos patogénicos y el estrés oxidativo, encontraron que en $S$. minor los niveles de $\beta$-caroteno son dependientes del estrés oxidativo causado en el momento de diferenciación de los esclerocios por peroxidación de lípidos y proteínas en micelio diferenciado a partir de aquéllos; así, los niveles de $\beta$-caroteno fueron 2,5 veces mayores en los micelios diferenciados que en los no diferenciados.

\section{Patogénesis}

La patogénesis, que considera el origen y el ciclo de la enfermedad, incluye las diferentes fases de inoculación, la penetración, el establecimiento de la infección y la colonización del patógeno (invasión, crecimiento, reproducción, dispersión y supervivencia del patógeno). La patogénesis, como proceso dinámico y complejo, involucra la capacidad inherente del patógeno y los múltiples factores que gobiernan la penetración e infección de la planta hospedera, que a su vez posee una serie de mecanismos de defensa que deben ser rotos, inactivados o anulados, antes de que la enfermedad pueda desarrollarse. Esta interacción entre el huésped y el patógeno está condicionada por las condiciones ambientales y por el tiempo de la relación (Lumsden, 1979). Finalmente, el grado de la infección como tal, es la resultante de la interacción de todos los elementos de un patosistema que involucra el cultivo, el patógeno, el ambiente, las prácticas agrícolas, los agentes bióticos relacionados en algunos casos, etc. (Maiorano et al., 2009).

\section{Inoculación}

La inoculación o contacto del patógeno con el huésped se presenta de dos formas. La primera, miceliogénica, a través de las hifas provenientes del esclerocio original que alcanzan la base del tallo de la lechuga, las hojas bajeras y el sistema radical. La segunda forma, carpogénica, se presenta a través de las ascosporas que se producen en los apotecios generados a partir de las hifas provenientes de los esclerocios, cuando las condiciones ambientales permiten este desarrollo. Esta segunda forma de inoculación es masiva y obliga a desarrollar estrategias de control diferentes a las primeras. La forma carpogénica permite el avance rápido de la enfermedad por la lluvia continua de esporas (Clarkson et al., 2003) provenientes del mismo campo o de otros vecinos como se ha demostrado en estudios realizados por Hammond et al. (2008).

\section{Penetración al huésped}

En el caso de Sclerotinia spp., la habilidad para penetrar e infectar el tejido del huésped depende del tipo de inóculo, las particularidades del huésped, el nivel de nutrientes del hongo y de las condiciones ambientales (Subbarao, 1998; Lumsden, 1979).

De Bary (1886) observó que Sclerotinia requiere nutrientes externos para una infección exitosa. Lumsden (1979) mostró que las hifas de Sclerotinia penetraban sólo después de haber sido "alimentadas y desarrolladas". Así, la penetración ocurría cuando las hifas eran colocadas en una gota de solución de nutrientes en el huésped. En el caso de ascosporas colocadas en agua, aunque germinaban eran incapaces de formar apresorios en la superficie del huésped y penetrar posteriormente (De Bary, 1887).

Aunque Abawi et al. (1975) sostenían que en fríjol, el inóculo requiere para su nutrición de una fuente de materia orgánica como requisito para la penetración del huésped, tanto para las as- 
cosporas como para los esclerocios germinados. Adams y Tate (1976) afirmaban que la infección de plantas de lechuga por $S$. minor ocurría en ausencia de materia orgánica disponible porque la masa de micelio aparentemente tiene suficientes nutrientes de reserva para permitir la penetración directa.

Aunque existen reportes de la entrada de tubos germinativos del micelio de $S$. sclerotiorum en hojas de papa a través de estomas (Jones, 1976), la penetración generalmente ocurre directamente a través de la cutícula de las hojas. La actividad lipolítica encontrada por Tariq y Jeffries (1987) sólo se dio en las hojas de fríjol, en el sitio de contacto entre las vesículas del apresorio y la cutícula y posteriormente en el poro de penetración en el tejido foliar, sin embargo no se ha observado actividad lipolítica en la invasión al tejido del tallo.

Cuando hay germinación carpogénica se forman los apresorios, los cuales son estructuras complejas, multicelulares, cuya formación requiere estímulos de contacto (De Bary, 1887). La complejidad del tipo de apresorios está relacionada directamente con la superficie del huésped y es por ello que varían dependiendo de la dificultad para penetrar, mostrando con ello que en el proceso de infección no existe un patrón definido como tal y ello podría explicar la capacidad de infectar gran número de plantas (Tariq y Jeffries, 1984).

Una vez en contacto con la cutícula del huésped, las hifas se bifurcan en dos estructuras en forma de dedo y eventualmente desarrollan cojines infecciosos en forma de domo (Lumsden, 1979). Los cojines infecciosos se adhieren estrechamente a la superficie del huésped por medio de un material mucilaginoso (Boyle, 1921) que mancha el material circundante con una coloración oscura (Prior y Owen, 1963; Lumsden y Dow, 1973); aparentemente, este material mucilaginoso y la forma de domo del cojín le permiten al mismo ejercer una considerable fuerza en la cutícula para ingresar mecánicamente al tejido del huésped (Abawi et al., 1975; Boyle, 1921; Lums- den y Dow, 1973). En S. minor el tipo de apresorio formado dependerá de la resistencia de la superficie a penetrar, con lo cual no se presenta un tipo único del mismo sino que variaría (Tariq y Jeffries, 1984).

\section{Infección del huésped}

Después de la penetración de la cutícula del huésped se forma una especie de vesícula granular inflada entre la cutícula y la epidermis (Boyle, 1921; Lumsden, 1979). Esta vesícula permite que la hifa de infección se desarrolle gradualmente desde los cojines de infección e invada los tejidos del huésped, y de acuerdo con De Bary (1887), indiscriminada, es decir que ocurre entre y a través de las células.

En tejidos de frijol infectados por S. sclerotiorum o $S$. minor, las hifas de infección crecen radialmente desde las vesículas y se desarrollan entre la cutícula y la capa de células epidermales e intercelularmente en la corteza (Lumsden y Dow, 1973).

Las hifas de infección indudablemente son las responsables del rompimiento de las defensas del huésped, de la colonización inicial de los tejidos (Boyle, 1921; Lumsden y Dow, 1973) y probablemente de los cambios en los tejidos infectados del huésped y además estarían asociadas con el avance de las márgenes de las lesiones en el huésped.

Entre los cambios en los tejidos ocasionados por las hifas de infección, se mencionan las alteraciones en los materiales pécticos de las paredes celulares, la muerte de las células, la acumulación de fluidos y agua en las márgenes de avance, los cambios en la permeabilidad de las células en avance y la producción de enzimas y otras sustancias responsables de la patogenicidad (Lumsden, 1979).

\section{Colonización del huésped}

Las hifas ramificadas invaden las células y espacios intercelulares en la corteza. Ellas también están asociadas con la destrucción de la estruc- 
tura cristalina de la pared celular del huésped (Calonge et al., 1969).

Después de la extensiva colonización del tejido, las hifas ramificadas emergen de los tejidos del huésped, primero a través de los estomas o a través de la cutícula (Lumsden y Dow, 1973).

La penetración exclusivamente intercelular de las hifas de infección a través del tejido (Lumsden y Dow, 1973) es incrementada por enzimas pectolíticas producidas por Sclerotinia spp., capaces de degradar la lamela media de las células del huésped; dichas enzimas, endo y exopectinasa, celulasas, hemicelulasas y proteasas, se cree que facilitan la degradación de la pared celular y la colonización posterior. Lumsden (1979) reporta que también otros autores coinciden en el hecho de que las enzimas pectolíticas siempre están asociadas con las enfermedades causadas por Sclerotinia. Finalmente, el conjunto de hifas entrelazadas forman un micelio algodonoso en la superficie de las lesiones maduras.

\section{Requerimientos de nutrientes para la infección}

La nutrición de Sclerotinia spp. durante todos los estados del desarrollo de la enfermedad es probablemente el factor más importante en la determinación del éxito o fracaso en el establecimiento de la enfermedad en el huésped (Lumsden, 1969, 1979).

Durante la infección, el hongo se organiza en hifas especializadas de infección, que requieren una cantidad considerable de energía y por tanto una abundante cantidad de nutrientes disponibles. Así, la nutrición dada por una determinada base alimenticia puede determinar si ocurre o no la enfermedad en un huésped potencial. Algunas enzimas como la celulasa, la hemicelulasa, la poligalacturonasa, la fosfatidasa, las enzimas proteolíticas y otras enzimas pueden cumplir un papel nutricional en la patogénesis. La acción de estas enzimas en las paredes y los contenidos celulares pueden proporcionar un suministro abundante de carbono y nitrógeno, esencial para la intensa actividad metabólica de Sclerotinia spp., a medida que las hifas de infección se mueven rápidamente a través de los tejidos del huésped y podrían ser responsables de la degradación de la pared celular y así volver disponibles abundantes carbohidratos y el nitrógeno, este último por la acción de la fosfatidasa y las proteasas.

\section{El papel del ácido oxálico en la patogénesis}

De Bary (1886) observó una asociación entre la producción de ácido oxálico en zanahoria y la infección por S. sclerotiorum. Otros autores igualmente reportan al ácido oxálico como una toxina producida por $S$. sclerotiorum en girasol y otros cultivos. Así mismo, Noyes y Hancock (1981), Godoy et al. (1990) y Cessna et al. (2000) afirman que la secreción de ácido oxálico por $S$. sclerotiorum podría ser un determinante esencial de su patogenicidad. En girasol se encontró una correlación entre la severidad de la enfermedad, la acumulación de ácido oxálico, la disminución del pH y la inhibición de la polifenoloxidasa; se concluyó que existe una estrecha relación entre la virulencia de $S$. sclerotiorum y la producción de ácido oxálico, lo mismo que su efecto en el metabolismo fenólico del huésped al inhibir la polifenoloxidasa (Magro et al., 1984). En S. sclerotiorum, la secreción de poligalacturonasa y la reducción del pH por el ácido oxálico son determinantes en la patogenicidad del hongo (Cotton et al., 2003).

De igual manera se encontró una alta correlación entre la degradación del ácido oxálico y el estímulo de la producción de la enzima $\beta$-1,3-gluconasa utilizando el micoparásito Coniothyrium minitans lo cual muestra que el biocontrol con aquél está fundamentado en la degradación del ácido oxálico (Ren et al., 2007). La evidencia para tal afirmación parece basarse en la recuperación de concentraciones milimolares de oxalato de tejidos infectados (Bateman y Beer, 1965; Godoy et al., 1990), y adicionalmente porque la 
inyección manual de oxalato en plantas, o de un filtrado de un cultivo que contenía oxalato, desarrolló los síntomas de una enfermedad como Sclerotinia, independiente del patógeno (Bateman y Beer, 1965; Noyes y Hancock, 1981).

Tal evidencia ha sido fortalecida por la observación de que mutantes de Sclerotinia que son deficientes en la habilidad para sintetizar oxalato, no son patogénicos, a pesar de poseer todo el complejo de enzimas degradativas, incluyendo la poligalacturonasa, pectin metilesterasa y celulasa; mientras que los aislamientos que mantienen su capacidad de biosintetizar oxalato exhiben virulencia normal (Godoyet al., 1990; Subbarao, 1998).

De acuerdo con Cessna et al. (2000), S. sclerotiorum es miembro de un grupo de al menos 12 hongos patógenos de plantas que generan y secretan concentraciones milimolares de oxalato a su alrededor.

La especulación acerca del mecanismo o mecanismos por los cuales la secreción de oxalato podría incrementar la virulencia de Sclerotinia se centra actualmente en tres modos de acción:

- A causa de que varias de las enzimas secretadas por el hongo durante la invasión de los tejidos huéspedes (ej. poligalacturonasa) tienen su máxima actividad a $\mathrm{pH}$ bajos; varios investigadores han postulado que el oxalato podría aumentar la virulencia de Sclerotinia por un cambio en el pH del apoplasto, a un valor más adecuado para la degradación enzimática de la pared celular de la planta (Bateman y Beer, 1965).

- Debido a que el oxalato puede ser tóxico para las plantas hospederas, presumiblemente a causa de su acidez, se ha sugerido que la secreción de oxalato debilita la planta facilitando así la invasión (Noyes y Hancock, 1981).

- La quelación del $\mathrm{Ca}^{2+}$ de la pared celular por el oxalato compromete la función del calcio, del cual dependen las respuestas de defensa y debilita la pared celular de la planta (Bateman y Beer, 1965).

Pero las plantas también responden a la invasión de un hongo patogénico. De acuerdo con Cessna et al. (2000), una de las primeras respuestas de resistencia por parte de los tejidos infectados de la planta contra la invasión de un microbio es el rompimiento oxidativo, el cual consiste en

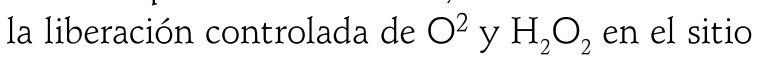
de ingreso del patógeno. Sin embargo, el rompimiento oxidativo es inhibido a bajos $\mathrm{pH}$ y requiere un incremento en el $\mathrm{Ca}^{2+}$ (Cessna et al., 2000).

El ácido oxálico producido por el patógeno afecta el pH de los tejidos infectados (Lumsden, 1979) reduciéndolo (Subbarao, 1998) de 5,0 a 4,0 (Lumsden, 1972) mediante la liberación de oxalato (Cessna et al., 2000) y disminuyendo así la viabilidad celular de los tejidos y, por lo tanto, la capacidad de la planta de responder a la invasión del patógeno (Subbarao, 1998).

El efecto del incremento de la acidez en el desarrollo de las lesiones favorecería la actividad de las enzimas pectolíticas, así como de las celulasas, hemicelulasas y otras enzimas hidrolíticas (Lumsden, 1979) e inhibiría la acción de enzimas tales como o-difenol-oxidasa, la cual está implicada en los mecanismos de defensa del huésped; así, la inhibición de aquella enzima incrementa la patogenicidad de S. sclerotiorum (Ferrar y Walker, 1993).

La colonización del huésped por Sclerotinia requiere la secreción del ácido oxálico que a su vez suprime la actividad del oxígeno reactivo (Walz et al., 2008). Durante el proceso de la infección, el patógeno secreta grandes cantidades de ácido oxálico que facilitan dicho proceso. Por otro lado, las enzimas oxalato oxidasa y oxalato decarboxilasa pueden detoxificar el ácido oxálico para convertirlo en $\mathrm{CO}_{2}$ y $\mathrm{H}_{2} \mathrm{O}_{2}$ (Donaldson et al., 2001; Hu et al., 2003; Walz et al., 2008). 
Lumsden (1979) afirma que el efecto del ácido oxálico en los tejidos enfermos podría ser múltiple. Así, durante los estados iniciales de desarrollo de la enfermedad y avance de la margen de la lesión, el ácido oxálico podría trabajar de forma sinérgica con las enzimas pectolíticas, tal como sucede en otras enfermedades.

El ácido oxálico puede ser responsable también de los síntomas de marchitez (Noyes y Hancock, 1981), asociados con las enfermedades causadas por Sclerotinia spp. (Lumsden, 1979), puesto que los cristales identificados como oxalato han sido observados ocluyendo los vasos del xilema (Lumsden y Dow, 1973; Noyes y Hancock, 1981).

\section{Síntomatología}

De acuerdo con Subbarao (1988), la pudrición blanda de la lechuga generalmente se manifiesta en dos etapas de desarrollo de la planta. Entre tres y cuatro semanas después de la emergencia de la plántula, caso poco frecuente, o cuando la planta está cercana o en la madurez, lo cual es lo más común (Adams y Tate, 1975; Abawi y Grogan, 1979; Subbarao, 1998; Koike y Davis, 2009). Bajo condiciones de Cota (Cundinamarca) y dependiendo del inóculo, se presenta un periodo de incubación de 3 a $4 \mathrm{~d}$ donde se hacen evidentes los síntomas y luego uno de latencia de 4 a 5 d en S. minor y $S$. sclerotiorum respectivamente, a partir de los cuales se forman micelio algodonoso y esclerocios (Smith, 2007).

Adams y Tate (1975) y Abawi y Grogan (1979) reportan también la etapa posterior a la formación de la cabeza como un periodo en el cual se hace más evidente la enfermedad.

Varios autores coinciden en que la infección se inicia en las hojas más viejas, cerca de la superficie del suelo (Abawi y Grogan, 1979; Walker, 1959; Purdy, 1979; Ferreira y Boley, 1992).

El síntoma inicial de la pudrición blanda de la lechuga es una marchitez de las hojas exteriores de la planta que dan a la misma una apariencia de estrés (Walker, 1959; Purdy, 1979; Subbarao, 1998).

Gradualmente las hojas exteriores son infectadas, se marchitan y caen sobre el suelo, mientras que las hojas de la cabeza permanecen erectas (Purdy, 1979; Abawi et al., 1985; Subbarao et al., 1997). A medida que el hongo coloniza el tejido huésped produce unas lesiones de color café pálido (Koike y Davis, 2009).

Posteriormente, hay una pudrición blanda de la cabeza y las hojas de la planta (Purdy, 1979; Abawi et al., 1985; Bell et al., 1998; Subbarao et al., 1998; Laemmlen, 2002), la cual presenta finalmente el aspecto de una masa gelatinosa $\mathrm{o}$ viscosa (Ávila de Moreno et al., 1992).

Subsecuentemente, bajo condiciones húmedas (Abawi y Grogan, 1979; Subbarao, 1998), se forma un micelio blanco algodonoso y se producen los esclerocios (Walker, 1959; Abawi y Grogan, 1979; Purdy, 1979; Koike y Davis, 2009; Laemmlen, 2002) sobre, dentro y alrededor de las plantas infectadas (Walker, 1959; Purdy, 1979).

Según Messiaen y Lafon (1968) los esclerocios son inicialmente blancos, del color del micelio que los origina, después toman una coloración grisácea y finalmente se tornan negros por completo.

Abawi y Grogan (1979) reportan un trabajo de Marcum y Grogan (no publicado) en el cual la muerte de la planta puede ocurrir a la semana siguiente de la infección de las hojas bajeras, en un periodo de 7 a $14 \mathrm{~d}$ cuando la infección es a la raíz principal, mientras que cuando la infección se origina en raíces secundarias, el progreso de la enfermedad es más lento y se requiere un periodo de incubación de 3 semanas o más para la expresión de los síntomas y la muerte de la planta.

Existe una diferencia entre la sintomatología de las infecciones causadas por ascosporas y las causadas por esclerocios; es decir entre la infección 
por S. sclerotiorum y S. minor. Subbarao (1998) afirma que cuando los esclerocios causan la infección, los síntomas iniciales se presentan en las hojas inferiores y la infección avanza hacia capas sucesivas de hojas; mientras que, cuando las ascosporas inician la infección, los síntomas se originan en las áreas expuestas de las plantas de lechuga, donde las ascosporas caen y causan la infección. Debido a que $S$. sclerotiorurm produce esporas aéreas, es común observar los síntomas en las partes aéreas de la planta como en la cabeza de la lechuga (Laemmlen, 2002), mientras que $S$. minor usualmente ataca el tallo en, o cerca de la superficie del suelo.

El tipo de enfermedad causada por S. sclerotiorum es determinada por el modo de "germinación" del inóculo. Así, cuando la germinación es miceliogénica se presenta pudrición radical, pudrición de la base del tallo y marchitez; mientras que cuando la germinación es carpogénica se presenta pudrición de la cabeza o pudrición de la vaina en fríjol (Huang et al., 1998). En cultivos como el girasol, el fríjol, el cártamo y la canola las ascosporas constituyen la principal fuente de infección de pudriciones en los campos comerciales; pero en la medida que se reduzca el número de esclerocios en el campo se reducirá el número de apotecios productores de ascosporas (Huang et al., 2006). Así mismo, las plantas infectadas por ascosporas aéreas forman esclerocios en las partes aéreas (Subbarao, 1998).

En conclusión, S. sclerotiorum y S. minor son patógenos que poseen una gran capacidad infectiva, por atacar numerosos cultivos de importancia agrícola y que, dadas las características originadas en la forma reproductiva, representan una seria amenaza para los cultivos hortícolas de la Sabana de Bogotá. En los cultivos de lechuga se ha presentado la infección a través de esclerocios en mayor medida en comparación con la forma carpogénica, la cual se presenta solo con S. sclerotiorum en condiciones de alta humedad relativa y bajas temperaturas permanentes, como se comprobó en el trabajo realizado por Arias y Tautiva (2006) realizado en el municipio de Mosquera. Con respecto a los dos patógenos, es posible distinguirlos por el tipo de infección, el tamaño de los esclerocios y a través de pruebas de laboratorio. Las medidas de control deberán dirigirse a reducir la oferta de esclerocios, a través de medidas de rotación de cultivos tolerantes y el uso de micoparásitos.

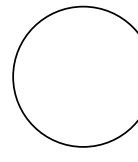

\section{REFERENCIAS BIBLIOGRÁFICAS}

Abawi, G.S. y R.G. Grogan. 1979. Epidemiology of diseases caused by Sclerotinia species. Phytopathol. 69, 899-904.

Abawi, G.S.; F.J. Polach y W.T. MOlin. 1975. Infection of bean by ascospores of Whetzelinia sclerotiorum. Phytpathol. 65, 673-678.

Adams, P.B. y E.A. Ayers. 1979. Ecology of Sclerotinia species. Phytopathol. 69, 896-898.

Adams, P.B. y C.J Tate. 1976. Mycelial germination of sclerotia of Sclerotinia sclerotiorum on soil. Plant Dis. Reporter 60, 515-518.
Agrios, G.N. 2005. Plant pathology. Elsevier Academic Press, San Diego, CA.

Arias, A.y A. Tautiva. 2006. Evaluación de tres métodos de control para el manejo de Sclerotinia (Sclerotinia sclerotiorum). Trabajo de grado. Facultad de Agronomía, Universidad Nacional de Colombia, Bogotá

Ávila de Moreno, C.; J. Velandia y A. López. 1992. Enfermedades de algunas especies hortícolas y su manejo. pp. 93-116. En: Memorias Primer Curso Nacional de Hortalizas de Clima Frío. ICA, Mosquera, Colombia.

Ávila de Moreno, C.; J. Velandia y A. López, A. 1996. Enfermedades y plagas de las hortalizas y su manejo. 
ICA, División de Sanidad Vegetal, Unidad Proyectos de Prevención, Bogotá. pp. 13-17.

Bateman, D.F. y S.V. Beer. 1965. Simultaneous production and synergistic action of oxalic acid and polygalacturonase during pathogenesis of Sclerotium rolfsii. Phytopathol. 55, 204-211.

Bell, A.A.; L. Liu; B. Reidy; R.M. Davis y K.V. Subbarao. 1998. Mechanisms of subsurface drip irrigation mediated suppression of lettuce drop caused by Sclerotinia minor. Phytopathol. 88, 252-259.

Ben-Yephet, Y.; A. Genizi y E. Siti. 1993. Sclerotial survival and apothecial production by Sclerotinia sclerotiorum following outbreaks of lettuce drop. Phytopathol. 83, 509-513.

Boyle, C. 1921. Sudies in the physiology of parasitism. VI. Infection by Sclerotinia libertiana. Ann. Bot. 35, 337-345

Calonge, F.D.; A.H. Fielding; R.J.W. Byrde y D.A. Akinrefon. 1969. Changes in ultrastructure following fungal invasion and the possible relevance. J. Exp. Bot. 20, 350-357.

Campos, T.; J. Rosello; M.D. Gomis; M.R. Hermosa; I. Grandona y E. Monte. 1998. Control biológico de la podredumbre húmeda de la lechuga, mediante la aplicación al agua de riego del hongo antagonista Trichoderma spp. Actas del XXX Congreso de la Sociedad Española de Agricultura Ecológica (SEAE). Valencia. En: www.agroecología.net/congresos/valencia/18.pdf; consulta: septiembre de 2009.

Casale, W.L. y L.P. Hart. 1986. Influence of four herbicides on carpogenic germination and apothecium development of Sclerotinia sclerotiorum. Phytopathol. 76, 980-984.

Cessna, S.G.; V.E., Sears; M.B. Dickman y P.S. Low. 2000. Oxalic acid, a pathogenicity factor for Sclerotinia sclerotiorum, supresses the oxidative burst of the host plant. Plant Cell 12, 1291-1299.

Chet, I.; H. Yigal y R. Mitchell. 1967. Chemical composition of hyphal and sclerotial walls of Sclerotium rolfsii. Can. J. Microbiol. 13(2), 137-41.

Clarkson J.P.; J. Staveley; K. Phelps; C.S. Young y J.M. Whipps. 2003. Ascospore release and survival in Sclerotinia sclerotiorum. Mycol. Res. 107(2), 213-222.

Cotton, P.; Z. Kasza; C. Bruel; C. Rascle y M. Fèvre. 2003. Ambient $\mathrm{pH}$ controls the expression of endopolygalacturonase genes in the necrotrophic fungus Sclerotinia sclerotiorum. FEMS Microbiol. Lett. 227, 163-169.

Cruickshank, R.H. 1983. Distinction between Sclerotinia species by their pectic zymograms. Trans. Brit. Mycol. Soc. 80 (1), 117-119
De Bary, A. 1886. Ueber einige Sclerotinien and Sclerotienkrankheiten. Bot. Zeitung 44, 377-474.

De Bary, A. 1887. Comparative morphology and biology of fungi. Mycetozoa and bacteria. Clerendon, London.

Dillard, H.R. y R.G. Grogan. 1985. Relationship between sclerotial spatial pattern and density of Sclerotinia minor and the incidence of lettuce drop. Phytopathol. 75, 90-94.

Donaldson, P.A.; T. Anderson; B.G. Lane; A.L. Davidson y D.H. Simmonds. 2001. Soybean plants expressing an active oligomeric oxalate oxidase from the wheat gf-2.8 (germin) gene are resistant to the oxalatesecreting pathogen Sclerotinia sclerotiorum. Physiol. Mol. Plant Pathol. 59, 297-307.

Erental, A., M.B. Dickman y O. Yarden. 2008. Sclerotial development in Sclerotinia sclerotiorum: awakening molecular analysis of a "Dormant" structure. Fungal Biol. Rev. 22(1), 6-16.

Favaron, F.; P. Alghisi y P. Marciano. 1992. Characterization of two Sclerotinia sclerotiorum polygalacturonases with different abilities to elicit glyceollin in soybean. Plant Sci. 83(1), 7-13.

Favaron, F.; P. Alghisi; P. Marciano y P. Magro. 1988. Polygalacturonase isoenzymes and oxalic acid produced by Sclerotinia sclerotiorum in soybean hypocotyls as elicitors of glyceollin. Physiol. Mol. Plant Pathol. 33(3), 385-395.

Ferrar, P.H. y J.R.L. Walter. 1993. o-Diphenol oxidase inhibition-an additional role for oxalic acid in the phytopathogenic arsenal of Sclerotinia sclerotiorum and Sclerotium rolfsii. Physiol. Mol. Plant Pathol. 43, 415-422.

Ferreira, S.A. y R.A. Boley. 1992. Sclerotinia sclerotiorum. Crop knowledge Master. En: www.extento.hawaii. edu/kbase/crop/Type/s_scler.htm; consulta: agosto de 2009.

Gil, R.; A. Smith,;B. Chaves; K. Wyckhuys; C. Forero y J. Jiménez. 2009. Eficiencia en la combinación de solarización y biofungicidas para el manejo de Sclerotinia spp. en lechuga (Lactuca sativa L.) Agron. Colomb. 27 (2), 193-201.

Godoy, G; J.R. Steadman; M.B. Dickman y R. Dam. 1990. Use of mutants to demonstrate the role of oxalic acid in pathogenicity of Sclerotinia sclerotiorum on Phaseolus vulgaris. Physiol. Mol. Plant Pathol. 37, 179-191.

Hammond, C.N.; T.F. Cummings y D.A. Johnson. 2008. Deposition of ascospores of Sclerotinia sclerotiorum. In and near potato fields and the potential to impact efficacy of a biocontrol agent in the Columbia Basin. Amer. J. Potato Res. 85(5), 353-360. 
Hu, X.; D.L. Bidney; N. Yalpani; J.P. Duvick; O. Crasta; O. Folkerts y G. Lu. 2003. Overexpression of a gene encoding hydrogen peroxide-generating oxalate oxidase evokes defense responses in sunflower. Plant Physiol. 133, 170-181.

Huang, H.C.; C. Chang y G.C. Kozub. 1998. Effect of temperature during sclerotial formation, sclerotial drynnes, and relative humidity on miceliogenic germination of sclerotia of Sclerotinia sclerotiorum. Can. J. Bot 76, 494-499.

Huang, H.C.; R.S. Erickson; L.M. Phillippe; C.A. Mueller; S.K. Sun y J.W. Huang. 2006. Control of apothecia of Sclerotinia sclerotiorum by soil amendment with $\mathrm{S}-\mathrm{H}$ mixture or Perlka ${ }^{\circledR}$ in bean, canola and wheat fields. Soil Biol. Biochem. 38(6), 1348-1352.

Imolehin, E.D.; R.G. Grogan y J.M. Duniway. 1980. Effect of temperature and moisture tension on growth, sclerotial production, germination and infection by Sclerotinia minor. Phytopathol. 70, 1153-1157.

Jones, D. 1976. Infection of plant tissue by Sclerotinia sclerotiorum: a scanning electron microscope study. Micron 7(4), 275-279.

Kohn, L.M. 1979. Delimitation of the economically important plant pathogenic Sclerotinia species. Phytopathol. $69,881-886$.

Koike, S.T. y R.M. Davis. 2009. Lettuce. Lettuce drop. UC IPM Pest management guidelines: Lettuce. UC ANR Publication 3450. University of California, Agriculture and Natural Resources, En: www.ipm. ucdavis.edu/PMG/r441100711.html; consulta: octubre de 2009.

Laemmlen, F. 2003. Sclerotinia diseases - Symptoms, signs and management. En: www.cesantabarbara.ucdavis.edu/ipm2.htm; consulta: septiembre de 2009.

Lumsden, R.D. 1979. Histology and physiology of pathogenesis in plant diseases caused by Sclerotinia species. Phytopathol. 69, 890-896.

Lumsden, R.D. y R.L. Dow. 1973. Histopathology of Sclerotinia sclerotiorum infection of bean. Phytopathol. 63, 708-715.

Magro, P.; P. Marciano y P. Di Lenna. 1984. Oxalic acid production and its role in pathogenesis of Sclerotinia sclerotiorum. FEMS Microbiol. Lett. 24(1), 9-12

Maiorano, A.; A. Reyneri; D. Sacco; A. Magni y C. Ramponi. 2009. A dynamic risk assessment model (FUMAgrain) of fumonisin synthesis by Fusarium verticillioides in maize grain in Italy. Crop Prot. 28(3), 243-256.

Marois, J.J. y P.B. Adams. 1985. Frequency distribution analyses of lettuce drop caused by Sclerotinia minor. Phytopathol. 75, 957-961.
Martínez-Pérez, Z.A. 2008. Algunos aspectos de la epidemiología del moho blanco de la lechuga (Lactuca sativa) en dos municipios productores de Cundinamarca. Trabajo de grado. Facultad de Ciencias, Pontificia Universidad Javeriana, Bogotá.

McLaren, D.L.; H.C. Huang y S.R. Rimmer. 1996. Control of aphotecial production of Sclerotinia sclerotiorum by Coniothyrium minitans and Talaromices flavus. Plant Dis. 80, 1373-1377.

Messianen, C.M. y R. Lafon. 1968. Sclerotinia y Botrytis. pp. 29-32. En: Enfermedades de las hortalizas. Ed. Oikos-tau S.A., Barcelona, España.

Nelson, D.; D.M. Hertsgaard y R.C. Holley. 1989. Disease progress of Sclerotinia wilt of sunflower at varying plant populations, inoculum densities, and enviroments. Phytopathol. 79, 1358-1363.

Noyes, R.D. y J.G. Hancock. 1981. Role of oxalic acid in the Sclerotinia sclerotiorum wilt of sunflower Helianthus annuus. Physiol. Plant Pathol. 18, 123-132.

Patsoukis, N. y C.D. Georgiou. 2007. Effect of thiol redox state modulators on oxidative stress and sclerotial differentiation of the phytopathogenic fungus $R$ hizoctonia solani. Arch. Microbiol. 188, 3, 205-297.

Patterson, C.L. y R.G. Grogan. 1988. Relationship of growth media and drying and of age of sclerotia to eruptive germination and infection by Sclerotinia minor. Plant Dis. 72, 1046-1048.

Prior, G.D. y J.H. Owen. 1963. Pathological anatomy of Sclerotinia trifolium on clover and alfalfa. Phytopathol. 54, 784-787.

Purdy, L.H. 1979. Sclerotinia sclerotiorum: History, diseases and symptomatology, host range, geographic distribution, and impact. Phytopathol. 69,875-880.

Ren, L.; G.Q. Li; Y.C. Han; D.H. Jiang y H.C. Huang. 2007. Degradation of oxalic acid by Coniothyrium minitans and its effects on production and activity of $\beta-1,3$-glucanase of this mycoparasite. Biol. Control 43(1), 1-11.

Smith, A. 2007. Caracterización, análisis especial y manejo integrado del moho blanco (Sclerotinia minor Jagger y S. sclerotiorum (Lib.) de Bary) en lechuga batavia (Lactuca sativa L. var. capitata) en la vereda La Moya (Cota-Cundinamarca). Trabajo de grado de Microbiólogo Agrícola y Veterinario. Facultad de Ciencias Básicas, Pontificia Universidad Javeriana, Bogotá.

Steadman, J.R. 1979. Control of plant diseases caused by Sclerotinia species. Phytopathol. 69, 904-907.

Subbarao, K.V. 1998. Progress toward integrated management of lettuce drop. Plant Dis. 82, 1068-1078. 
Subbarao, K.V.; S.T. Koike y J.C Hubbard. 1996. Effects of deep plowing on the distribution and density of Sclerotinia minor sclerotia and lettuce drop incidence. Plant Dis. 80, 28-33.

Subbarao, K.V.; J.C Hubbard y K.F Schulbach. 1997. Comparison of lettuce disease and yield under subsurface drip and furrow irrigation. Phytopathol. 87, 877-883.

Sun, P. y B. Yang. 2000. Light, temperature, and moisture effects on apothecium production of Sclerotinia sclerotiorum. Plant Dis. 84, 1287-1293.

Tariq, V.N. y P. Jeffries. 1984. Appresorium formation by Sclerotinia sclerotiorum: Scanning electron microscopy. Trans. Brit. Mycol. Soc. 82 (4), 645-651

Tariq, V.N. y P. Jeffries. 1987. Cytochemical localization of lipolytic enzyme activity during penetration of host tissues by Sclerotinia sclerotiorum. Physiol. Mol. Plant Pathol. 30(1), 77-91.

Trevethick, J.y R.C. Cooke. 1973. Non nutritional factors influencing sclerotium formation in some Sclerotinia and Sclerotium species. Trans. Brit. Mycol. Soc. 60 (3), 559-566.
Walker, J.C. 1957. Sclerotinia diseases of vegetable crops and field crops. pp. 365-370. En: Plant pathology. 2a ed. McGraw Hill, New York, NY.

Walker, J.C. 1959. Sclerotinia disease. pp. 218-219. En: Salvat, E. (ed). Enfermedades de las hortalizas. Barcelona, España.

Walz, A.I.; S. Zingen-Sell; S. Theisen y A. Kortekamp. 2008. Reactive oxygen intermediates and oxalic acid in the pathogenesis of the necrotrophic fungus Sclerotinia sclerotiorum. Eur. J. Plant Pathol. 120(4), 317-330

Willetts, H.J. y A.L. Wong. 1971. Ontogenetic diversity of sclerotia of Sclerotinia sclerotiorum and related species. Trans. Brit. Mycol. Soc. 57(3), 515-524.

Willetts, H.J. y S. Bullock. 1992. Developmental biology of sclerotia. Mycol. Res. 96(10), 801-816

Zervoudakis, G.; N. Tairis; G. Salahas y C.D. Georgiou. 2003. $\beta$-carotene production and sclerotial differentiation in Sclerotinia minor. Mycol. Res. 10(5), 624-631. 